\title{
Research on the Factory Automation Software Based on the SEMI Standard
}

\author{
Chenxi Wang ${ }^{1, a^{*}}$, Mingzhe Liu ${ }^{1, b}$, Aidong $\mathrm{Xu}^{1, \mathrm{c}}$ and $\mathrm{Ni} \mathrm{Jin}^{1, \mathrm{~d}}$ \\ ${ }^{1}$ Shenyang institute of Automation Chinese Academy of SciencesShenyang China \\ a wangchenxi@sia.cn, ${ }^{b} I m z @ s i a . c n,{ }^{c} x a d @ s i a . c n,{ }^{d}$ jinni@sia.cn
}

Keywords: IC equipment, SEMI, FA

Abstract. Factory automation (FA) system software is a bridge to realize information exchange between processing equipment of semiconductor equipment and factory host. It receives the control instruction sent by a host, and sends the control instruction to the manufacturing equipment after internal analysis and processing. At the same time, FA receives the real time data and the various process parameters sent by equipment, processes the data, and sends the data to the host. In this paper, analysis of the process of semiconductor processing equipment, according to the actual needs of the semiconductor processing equipment, research on FA Software based on the SEMI standard.

\section{Introduction}

At the beginning of semiconductor industry development, equipment manufacturers just focus on how to make the equipment normal, stable operation and achieve the requirements of manufacturing process. But with the rapid development of hardware design and technology, the requirements of manufacturers on quality of products and production efficiency increase fast. They want to use the automation equipment and device to replace the repeating the manual labor, to improve the quality of products, and the production efficiency.

At present, Semiconductor processing equipment abroad has been with the SECS/GEM communication interface, but few in the domestic. Therefore, developing the factory automation network system control software with independent intellectual property rights is more and more attention by the domestic manufacturers of semiconductor.

\section{SEMI standard in Semiconductor industrial automation process}

In the production process of a semiconductor integrated circuit, with the coordination of a variety of standard, we can process products on different equipment and different systems. The specific process is shown below.

The factory manufacturing execution system can track the unfinished product, and the host will determine the next process of the product, according to the program in the factory manufacturing execution system. The factory manufacturing execution system will sends commands to the transmission system, and transports the product to the destination device automatically. FA needs to provide the standard for host. The standard stipulates the behavior of transfer the carrier into the equipment or other internal buffers of the equipment, according to E87 (Carrier Management) and E84 (Enhanced Carrier Handoff Parallel I/O interface).

After the carrier is delivered to the device location, it reaches the loading device between transmission systems and equipment first. When the carrier is unloaded at the loading device, the equipment will read the carrier ID and the process information, submit to FA. FA will analyze the information and sent to the remote host. After the host verifies the information, the host will send processing command. In this process, FA needs consolidate the mechanical interface between production equipment and testing tools and automatic transmission system according to SEMI E15 (specification for tool load port). Through the mechanical motion of device, the system realizes the orientation of carrier. At the same time, FA needs to provide a common specification of concept, 
behavior and services for carrier ID according to SEMI E99 (the carrier ID reader/writer functional standard: specification of concepts, behavior, and services).

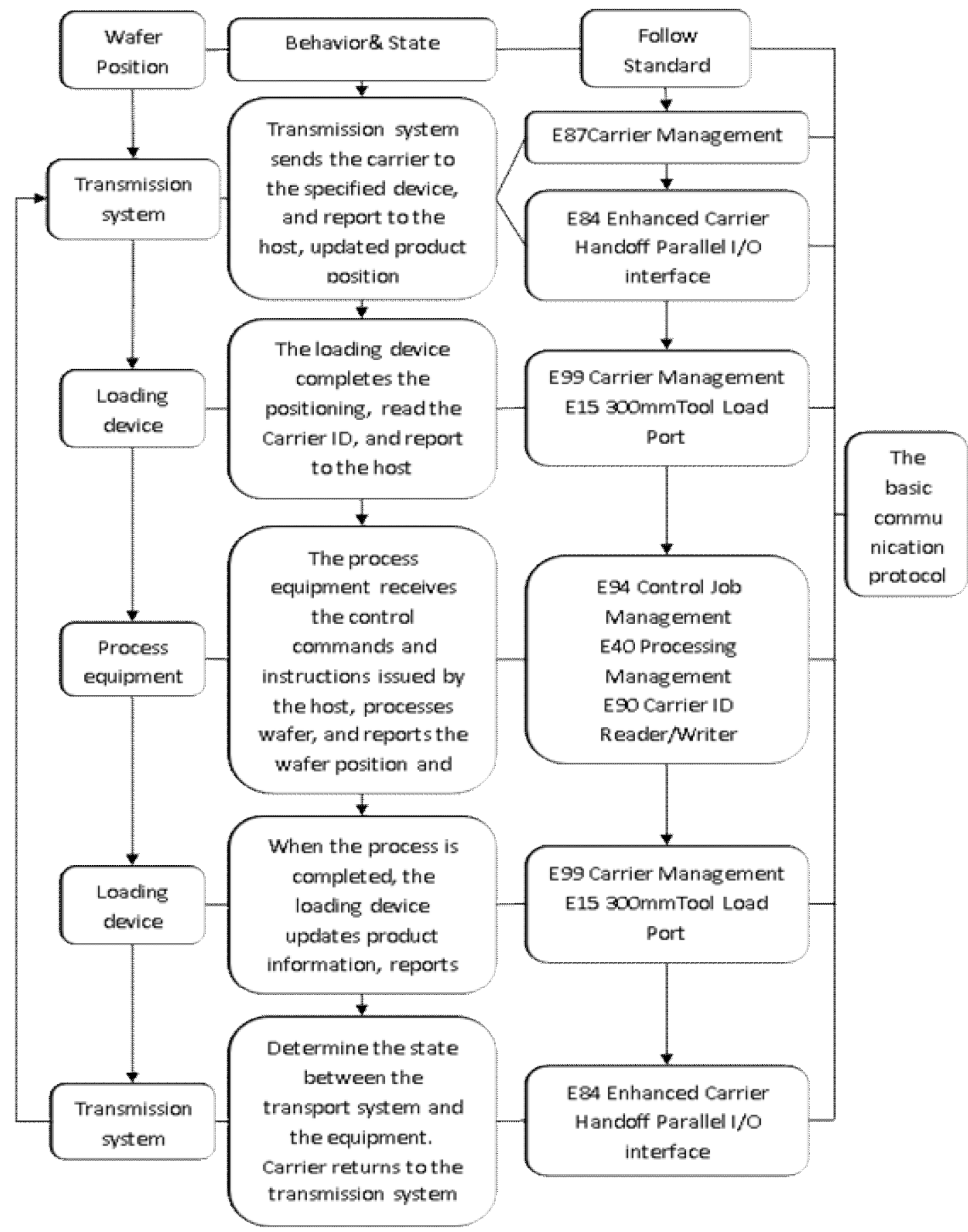

Fig1 Wafer Processing Flow Chart

After the products are transferred into the device, the host will issue control job and process job to the equipment through FA. In the process of semiconductor processing, the whole semiconductor manufacturing process is divided into one or more PROCESS JOB, the semiconductor processing equipment specified specific recipe of material processing for each PROCESS JOB. At the same time, define a CONTROL JOB says a work flow, each work process includes one or more PROCESS JOB, through the organization of the associated PROCESS JOBS, coordinate material deployment, unified management, batch processing completed work. FA makes the process job as an object, it defines the related attributes and service of process job according to the E40 (process management standard). FA also makes the control job as an object, defines the related attributes and service of control job, and organizes the process job, unified management according to the E94 (control job management). 
The above is only the introduction of some equipment control standard in semiconductor manufacturing process, in other respects, there are many widely used standard, for example, E42 (recipe management standard), E90 (substrate tracking), E95 (specification for human interface for semiconductor manufacturing equipment). These standards constitute the language of the semiconductor automation industry. They are used in each part of the industry. It makes that different equipment manufacturing, wafer fabrication, packaging and testing, and various ancillary facilities suppliers have common standards.

\section{Research on the factory automation software based on the SEMI standard}

As the bridge between the host and the device factory, FA can convey the instructions issued from the host to the equipment accurately and timely. At the same time, FA can feedback events occur on the device to factory host. Therefore, according to the actual demand of the software, FA is divided into several parts as follows: the underlying database module, the connection software module, the data analysis and processing module and the link ECS module.

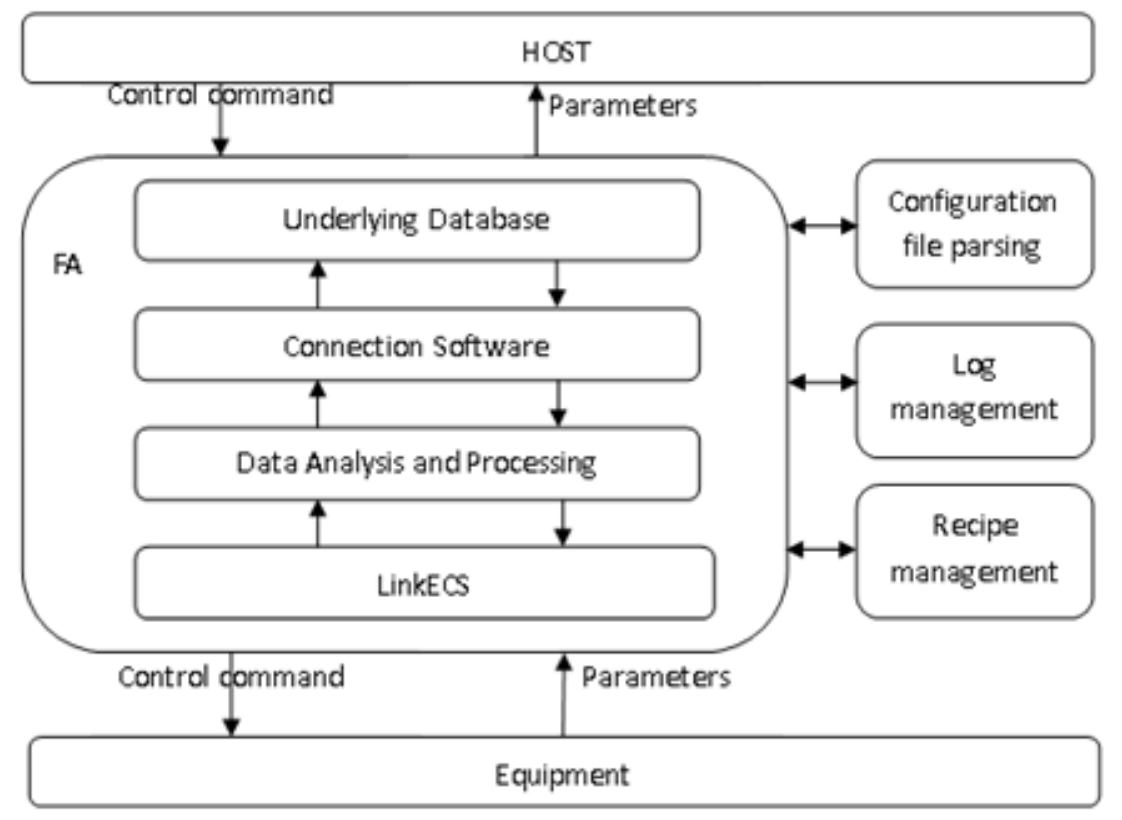

Fig2 FA Structure Diagram

The underlying database module realize the factory requirements of the SEMI standard, including E5, E37, E30, E39, E40, E94, E87, E90, E84 and other related standards. System calls the function provided by the underlying database module, transformations events conform to the SEMI standard, like $\mathrm{S}_{\mathrm{X}} \mathrm{F}_{\mathrm{Y}}$, and sends the events to the host. The host issues commands, such as start processing, pause processing, to the underlying database module with message form of $\mathrm{S}_{\mathrm{X}} \mathrm{F}_{\mathrm{Y}}$, and send to the ECS through the Link ECS module.

The LinkConX300 module is between the underlying database module and the data analysis processing module in the logical structure, it implements the data encapsulation and analysis. On the one hand, the LinkConX300 module changes information format, it makes that data processing module can identify the information. On the other hand, the LinkConX300 module reports the process parameters in equipment operation to the host with the form of $\mathrm{S}_{\mathrm{X}} \mathrm{F}_{\mathrm{Y}}$.

The data analysis and processing module is the core module of FA. After the data analysis and processing module analyzes the transmission of host, it sends the operating instruction to the equipment to control processing. The data analysis and processing module also Analysis and processes the parameters of equipment actual operation, and sends them to LinkConX300 module to package. At the same time, the data analysis and processing module visits recipe management module, to report or download recipes. The data analysis and processing module also visits log management module, to save the data to query for management. 
The Link ECS module is a communication module of FA. It is responsible for communication with the equipment. The Link ECS module gets the equipment event, and sends control commands and management commands to equipment.

In addition, there are some external service modules.

The recipe management module uses the framework of $\mathrm{C} / \mathrm{S}$, Including the recipe server and the recipe client. The recipe server receives data from the recipe client or the request to write, and reads recipe data from the recipe database. The recipe client communicates with the recipe server and $\mathrm{read} /$ write a recipe file. When FA receives processing recipe from control system, FA uses recipe client to get the recipe data from the recipe server according to the recipe ID, and analyzes recipe data, reports the recipe data to host at last.

The log management module is the same as the recipe management module, uses the framework of $\mathrm{C} / \mathrm{S}$, and including the log server and the log client. The log record module can be used by each module of the system, and the equipment management can query the log information by log function of GUI.

The configuration file management module manages all of the configuration files of FA, including the recipe format configuration files, the GCD files, the log files, and the SECSGEM files. The configuration file management module uses different methods to analyze configuration files according to different file types.

\section{Conclusion}

With the development of semiconductor industry, factory automation will play a more and more important role in the semiconductor industry in the future. In practical application, different understanding of SEMI standards often lead to the difference of the software, how to understand and use SEMI standard correctly will become an important research topic in the semiconductor industry. This paper presents SEMI material transport model of semiconductor integrated circuit production, analyses the basic process of material transportation and SEMI standard it follows, in factory automation. The model has been used in practical equipment, the normal operation of equipment proves the reliability of this method, and it is helpful for further research of semiconductor control system.

\section{Acknowledgments}

This paper is supported by the key laboratory of Chinese Academy of Sciences: Lab. Of Networked Control Systems, and thanks for it.

\section{References}

[1] J. van der Geer, J.A.J. Hanraads, R.A. Lupton, The art of writing a scientific article, J. Sci. Commun. 163 (2000) 51-59.

Reference to a book:

[2] W. Strunk Jr., E.B. White, The Elements of Style, third ed., Macmillan, New York, 1979.

Reference to a chapter in an edited book:

[3] G.R. Mettam, L.B. Adams, How to prepare an electronic version of your article, in: B.S. Jones, R.Z. Smith (Eds.), Introduction to the Electronic Age, E-Publishing Inc., New York, 1999, pp. 281-304.

[4] R.J. Ong, J.T. Dawley and P.G. Clem: submitted to Journal of Materials Research (2003)

[5] P.G. Clem, M. Rodriguez, J.A. Voigt and C.S. Ashley, U.S. Patent 6,231,666. (2001)

[6] Information on http://www.weld.labs.gov.cn 